\title{
The Bethlem and Maudsley Hospital item sheets (B-MIS)
}

\author{
The development and reliability of an instrument for routine collection of summary \\ clinical data
}

Jeremy TURK, Senior Registrar, Department of Psychological Medicine, Hospital for Sick Children, Great Ormond Street, London, WCIN 3JH (address for correspondence); NASSER F. LozA, Consultant Psychiatrist, Behman Hospital, Cairo, Egypt; JAN KASINSKI, Locum Senior Registrar, Department of Psychological Medicine, Hospital for Sick Children; RANDY KATZ, Honorary Lecturer, Institute of Psychiatry, De Crespigny Park, London, SE5; PETER McGuffin, Professor, Department of Psychological Medicine, University of Wales College of Medicine, Heath Park, Cardiff; and RoBIN MURRAY, Dean, Institute of Psychiatry, De Crespigny Park, London, SE5

The Bethlem and Maudsley Hospital item sheets (BMIS) are summaries in coded form which are completed for every patient admitted or readmitted to the joint hospitals. The Part 1 item sheet contains information which is included in the Part 1 case summary, as described in Notes on Eliciting and Recording Clinical Information, ${ }^{1}$ and is completed at the same time as the summary is prepared; that is, usually within a fortnight of the patient entering hospital. Similarly, the Part 2 item sheet contains information normally found in the discharge summary, and is completed at the same time that this summary is prepared; shortly after the patient has left hospital. The item sheets are restricted to pieces of information which can be readily coded by the registrar. Thus, of necessity, they concentrate on relatively 'hard' data.

The primary aim of the item sheets is to provide a comprehensive data base for research and for monitoring the work of the hospitals, which can be stored easily on computer, and rapidly accessed when needed. Coded data on in-patients of the joint hospitals have been collected in itemised form since the early 1950's, and have been used in a number of published studies. ${ }^{2.3}$

Earlier versions of the item sheets suffered from psychometric weaknesses. Some terms were felt to be ambiguous and the format was felt to be overcomplicated. Ratings recorded on a selected number of the items in an early version were found to be unreliable. ${ }^{4} \mathrm{~A}$ further study ${ }^{5}$ showed marked variability in rater agreement. The author attributed this finding to registrars tending to view the task of completing item sheets as an unnecessary burden, and hence often completing them in batches long after the patients had departed. Test-retest and inter-rater reliabilities for the individual items were never established. Also, it was not explicitly designed for computer assimilation. Hence, a full revision was undertaken, the goal being to produce them in a format that allowed coded data to be presented in readily storable and accessible form.

\section{Background to the present study}

A working party drafted lists of items with the aim of covering in easily codable format most of the 'hard' data contained in the admission and discharge clinical summaries. For ease of completion and coding it was aimed, where possible, to use a simple binary form i.e. present or absent. Thus, much 'soft' historical data had to be excluded.

A small-scale preliminary inter-rater reliability study on a series of 20 consecutive admissions to a ward of the Bethlem Royal hospital was undertaken by two of the authors (JK, PMCG). As a result, certain low reliability items were excluded. A glossary was prepared giving definitions, and directions, for each of the remaining items. Where possible, this drew heavily on authoritative sources, for example definitions found within the glossary of the Present State Examination. ${ }^{6}$ Following this it was decided that a more detailed reliability study should be undertaken.

\section{The study}

The medical records with accompanying admission and discharge summaries were obtained for two groups of consecutive admissions to acute adult psychiatric units. Table I shows the mean, standard deviation, and range for the patients' ages, and the sex distribution of the patients studied. Table II lists the 
TABLE I

Age mean, standard deviation, and range, and sex ratio, for patients studied

\begin{tabular}{|c|c|c|c|c|}
\hline Mean & $\begin{array}{c}\text { Age } \\
\text { Standard } \\
\text { Deviation }\end{array}$ & Range & Male & $\begin{array}{l}\text { Sex } \\
\text { Female }\end{array}$ \\
\hline 43.3 & 16.2 & $19-74$ & 11 & 19 \\
\hline
\end{tabular}

TABLE II

ICD-9 diagnoses of patients involved in study

\begin{tabular}{lc}
\hline \multicolumn{1}{c}{ Diagnosis } & $\begin{array}{c}\text { Number of } \\
\text { patients }\end{array}$ \\
\hline Schizophrenia & 11 \\
Manic depressive psychosis, depressed type & 7 \\
Manic depressive psychosis, manic type & 5 \\
Neurotic depression & 5 \\
Organic psychotic condition & 1 \\
Explosive personality & 1 \\
\hline Total & 30 \\
\hline
\end{tabular}

ICD-9 diagnoses for these individuals at discharge from hospital.

As the groups did not differ significantly on any of these variables the data were combined in the analysis of reliability.

Two of the authors (JT, NL) rated item sheets blind to each other. The resulting data were computer coded in binary form. Individuals were identified by code number only, thereby ensuring anonymity and maintaining confidentiality.

The accumulated data were analysed on the University of London Computer Centre AMDAHL computer, using a pre-existing programme. ${ }^{7}$ In most cases straightforward levels of agreement were assessed. For certain items, where one or other rater had given identical responses for all subjects, inter-rater agreement was calculated as a simple percentage, for statistical reasons.

The initial computer analysis yielded six items whose levels of inter-rater agreement were not significantly better than chance. The definitions of these items, as contained in the guide and glossary to the item sheets were scrutinised, and reasons for disagreements considered. Following mutually agreed revision of the definitions, these items were rerated for the 30 patients by JT and NL, again blind to each others ratings. This second analysis (Table III) left two items with persisting significant levels of dis- agreement; 'agitation' and 'anxiety-with autonomic symptoms'. These items are considered further in the discussion section of this paper.

\section{Comment}

The results of this study confirm the usefulness of the Bethlem Royal and Maudsley Hospital item sheets (B-MIS) in providing a reliable data collection system which can be completed in approximately five minutes per patient, by the junior psychiatrist responsible for that person.

The project emphasises the need for objective controlled evaluation of such tools designed for data storage. Despite extensive committee work and pilotting, there remained items (some of which represent extremely common psychiatric terms) for which reliability proved difficult to achieve. A project such as this allows for the refinement of such definitions, thereby improving their future usefulness, enhancing diagnostic precision, and allowing reliable operationalisation for research purposes.

The unreliability of the item 'agitated' was felt to have related to its particularly relaxed usage in the English language. The psychiatric term refers to observed overt behaviour, not merely a subjective feeling of tension or anxiety. The glossary has been amended to reflect this. It now emphasises that the term refers to a specific set of behaviours which reflect underlying anxiety.

The presence or absence of 'anxiety' was rarely disputed. Controversy centred around whether there were indeed accompanying autonomic symptoms, or whether they were subjective perceptions without physiological basis. In view of the good agreement as to which patients were anxious, and which were not, it was decided to create a unitary 'anxiety' term, thereby resolving the above dilemma.

A shortcoming of this study was the limited range of subjects, concentrating on patients with acute psychiatric disorders requiring in-patient management. There is a need to confirm the findings on larger samples of patients suffering from a variety of disorders, and covering the extremes of age. Indeed, the children's department of the Bethlem Royal and Maudsley hospitals has its own age-appropriate item sheets. ${ }^{8}$ It would seem logical and likely that item sheet rating systems would require tailoring in order to make them applicable to the population that is to be rated.

\section{Concluding remarks}

The Bethlem Royal and Maudsley hospital item sheets (B-MIS) appear to be a reliable means of rapidly and readily coding information pertaining to adult psychiatric in-patients, which can be easily 


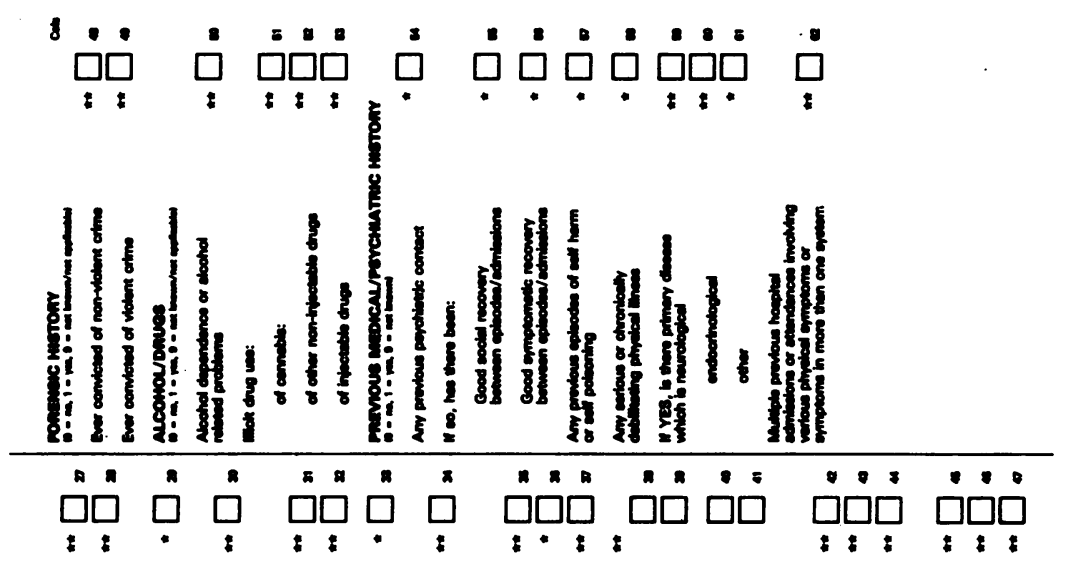

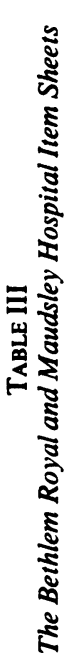
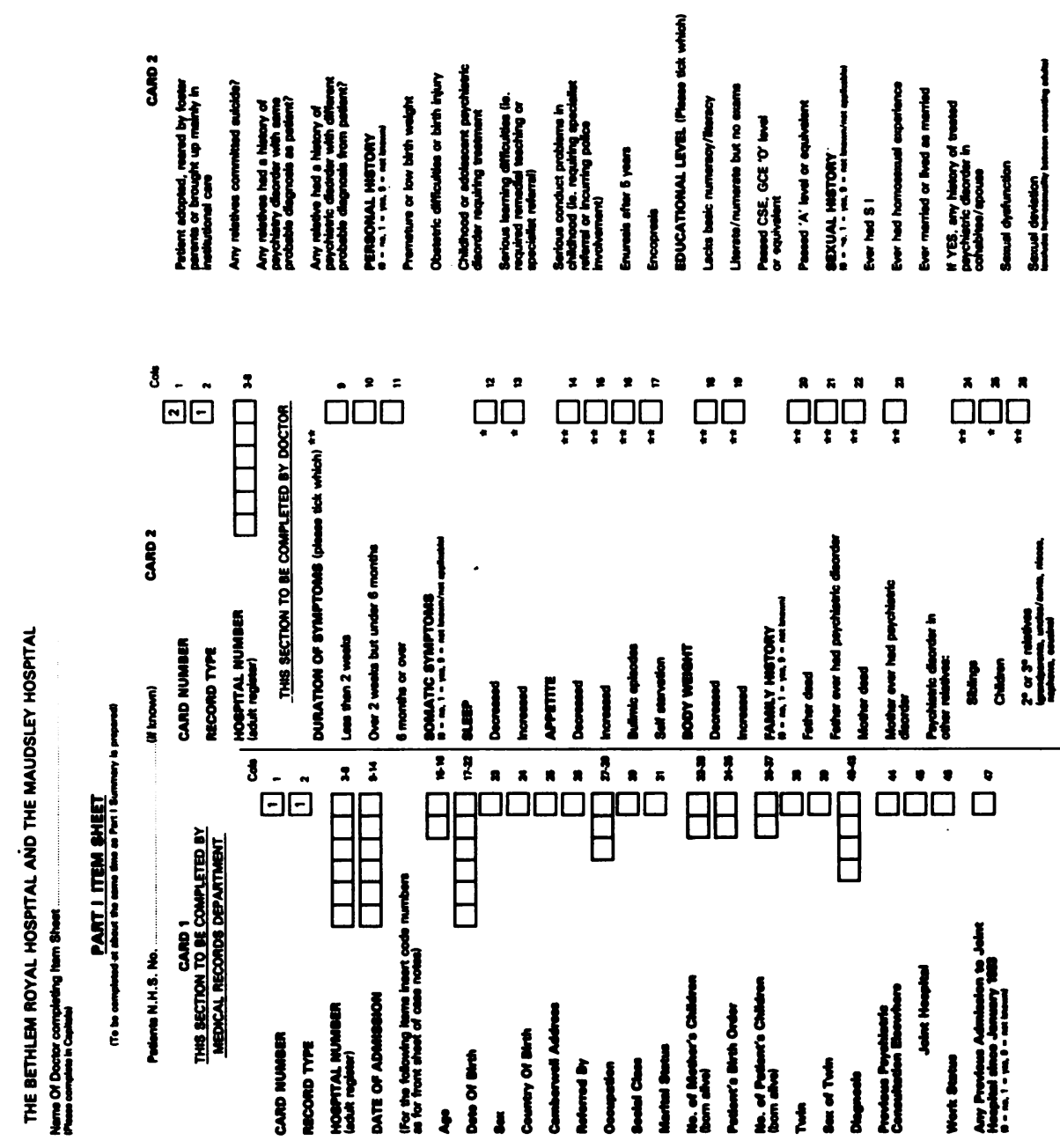
The Bethlem and Maudsley Hospital item sheets (B-MIS)
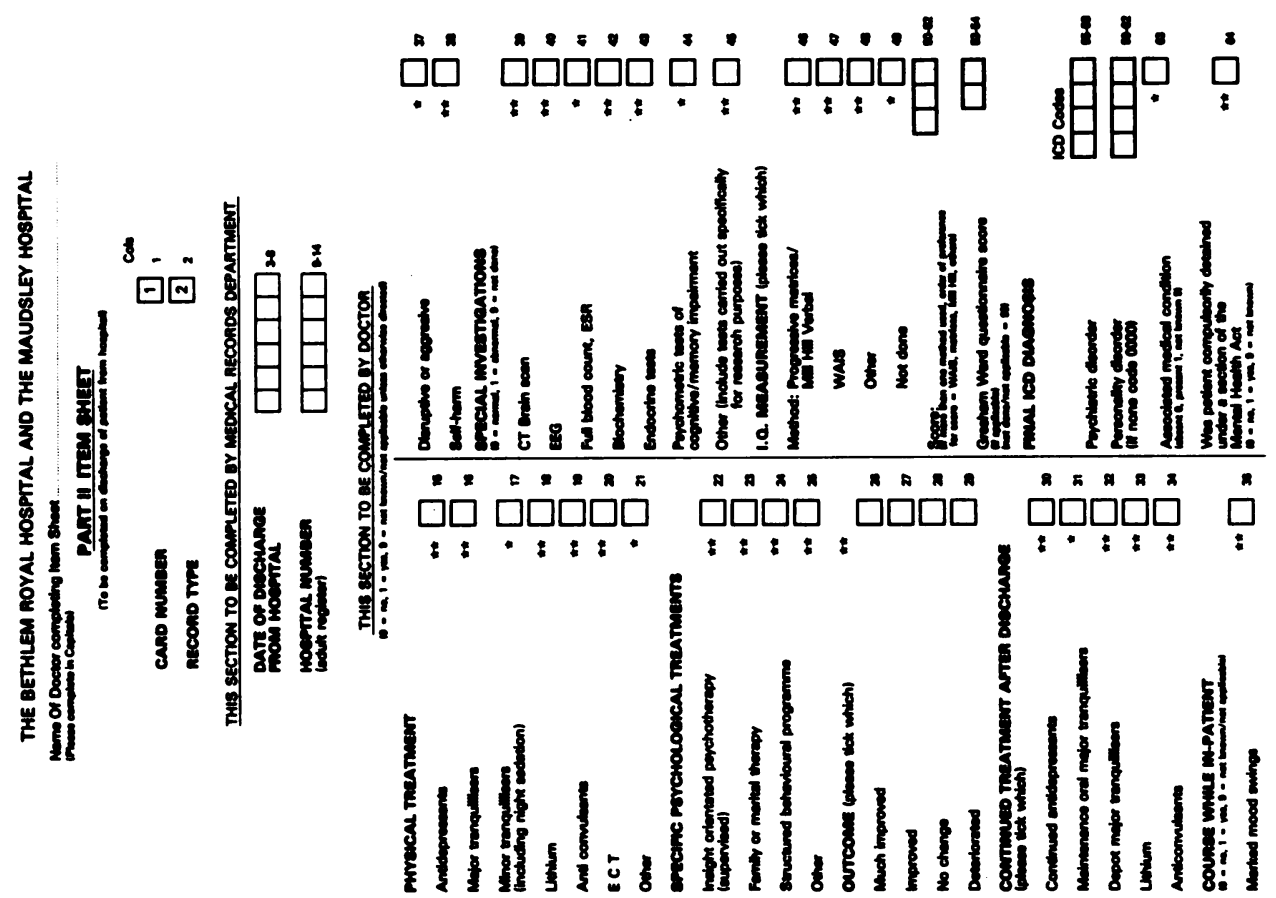

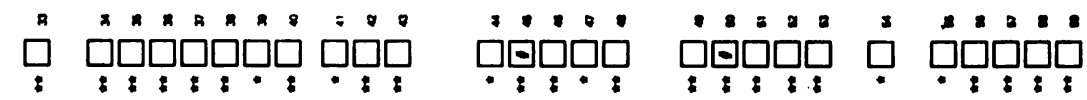

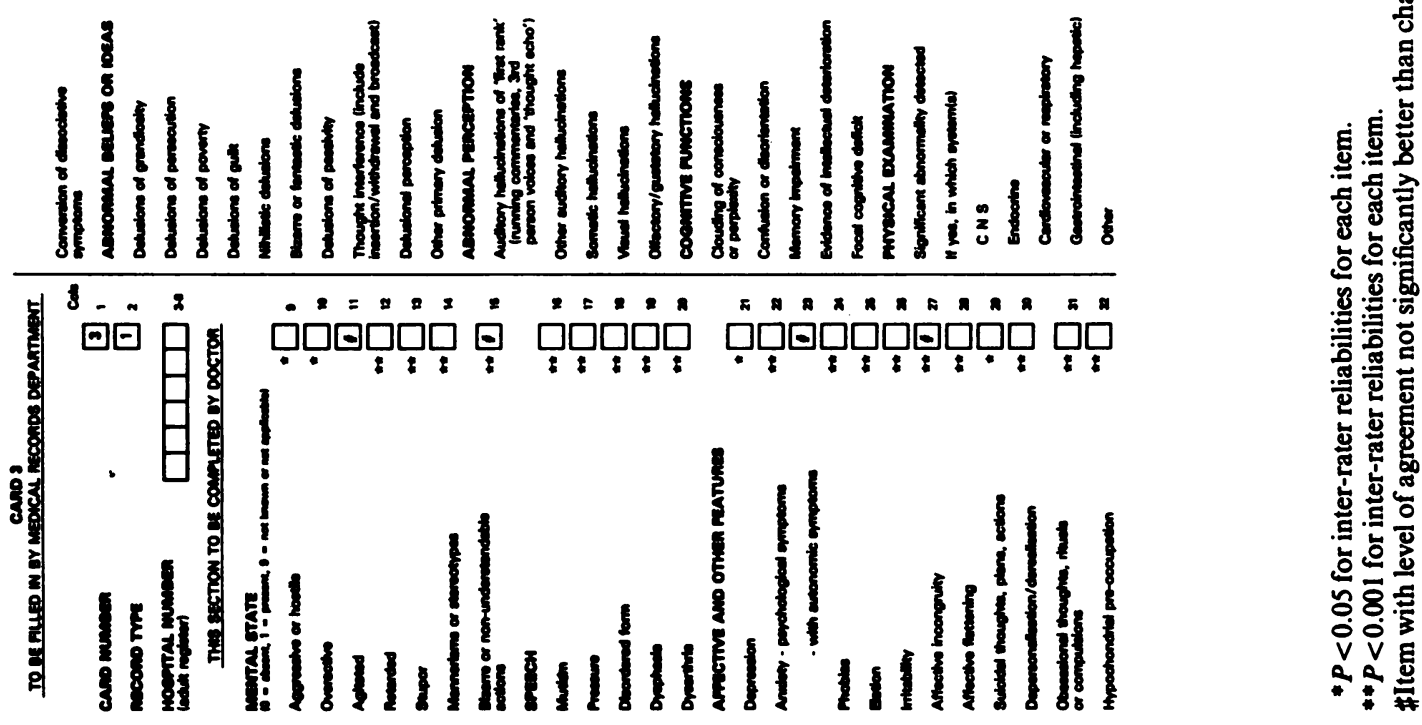


computerised, stored, and retrieved as required for research and administrative purposes. The need for objective scientific evaluation of such systems, and their constituent items, is emphasised by our findings. Such practice allows identification of poorreliability items, and subsequent revision of their definitions. The appropriateness of item sheets for the particular patient group under study always requires evaluation. Ultimately, the reliability and

\section{References}

${ }^{1}$ Hill, D., BiRley, J. L. T., Cawley, R. H., Kendell, R. E., Lishman, W. A., Post, F., Rutter, M., Wing, J. K. \& WolfF, H. H. (1973) Notes on Eliciting and Recording Clinical Information. Oxford University Press.

${ }^{2}$ Kendell, R. E. \& Gourlay, J. (1970) The clinical distinction between psychotic and neurotic depression. British Journal of Psychiatry, 117, 257-260.

${ }^{3}$ SHUR, E. (1982) Season of birth in high and low genetic risk schizophrenia. British Journal of Psychiatry, 140, 410-415.

${ }^{4}$ Grosz, H. J. \& Grossman, K. G. (1964) The sources of observer variation and bias in clinical judgments, Journal of Nervous and Mental Disease, 138, 105-113. usefulness of any data collected remains dependent on the skill and conscientiousness of the individual who completes the ratings.

This small-scale assessment study suggests that this approach to rapid coding of standardised information not only provides reliable data but also introduces a useful aide-memoire and an instructive intellectual training exercise for the aspiring psychiatrist in his everyday ward work.

5 (1968) The relationship between neurosis and psychosis, British Journal of Psychiatry, 114, 189-192.

${ }^{6}$ Wing, J. K., COOPER, J. E. \& SARTORIUS, N. (1974) The Measurement and Classification of Psychiatric Symptoms, London: Cambridge University Press.

${ }^{7}$ Ciccherti, D. V. (1978) Assessing observer and method variability in medicine. Connecticut Medicine, 42, 253-256.

${ }^{8}$ ThORLeY, G. (1982) The Bethlem Royal \& Maudsley Hospitals Clinical Data Register for Children and Adolescents. Journal of Adolescence, 5, 179-190.

\title{
A service for patients with Wilson's disease
}

\author{
T. R. Dening, Research Fellow, Department of Psychiatry; G. E. BerRios, Consultant \\ and University Lecturer in Psychiatry; and C. A. SEYMOUR, Consultant and University \\ Lecturer in Medicine, University of Cambridge Clinical School
}

Wilson's disease (WD) is an autosomal recessive disorder of copper metabolism with an incidence of about 30 per million (i.e. fewer than 2,000 in the UK). Nevertheless, it is important for two main reasons: its manifestations are protean and may lead it to present to a range of specialists; and its otherwise lethal course can be halted by treatment with chelating agents such as penicillamine and trientine. Published cases $^{1}$ and systematic study ${ }^{2}$ have shown that neuropsychiatric symptomatology is important in a high proportion. In fact, about one-fifth either present psychiatrically or are at least seen by a psychiatrist before WD is diagnosed.

Addenbrooke's Hospital, Cambridge has been the centre for the largest series of WD in the UK (over 200 cases) due to the presence of Dr John Walshe, who was responsible for the initial introduction of penicillamine $^{3}$ and other agents, as well as many clinical and laboratory studies of WD and copper metabolism. In September 1987 JMW retired, and the responsibility for WD passed to CAS. The aim of this paper is to describe the workings of the service until Autumn 1987, with particular respect to psychiatric aspects, and then to discuss future developments.

The service as operated by JMW, Reader in Metabolic Diseases and Consultant Physician, included clinical and laboratory components. All copper biochemistry was performed in a laboratory in the Department of Medicine. Junior medical staff were shared with the rest of the academic unit, but only involved with in-patients. All referrals, including 